\title{
Assimilation de données d'humidité des sols pour la prévision de crues : comparaison d'un modèle pluie-débit conceptuel et d'un modèle intégrant une interface sol-végétation-atmosphère Assimilation of soil moisture into hydrological models for flood forecasting: comparison of a conceptual rainfall-runoff model and a model with an explicit counterpart for soil moisture
}

\author{
A. Weisse, L. Oudin et C. Loumagne
}

Volume 16, numéro 2, 2003

URI : https://id.erudit.org/iderudit/705503ar

DOI : https://doi.org/10.7202/705503ar

\section{Aller au sommaire du numéro}

\section{Éditeur(s)}

Université du Québec - INRS-Eau, Terre et Environnement (INRS-ETE)

\section{ISSN}

0992-7158 (imprimé)

1718-8598 (numérique)

\section{Découvrir la revue}

Citer cet article

Weisse, A., Oudin, L. \& Loumagne, C. (2003). Assimilation de données d'humidité des sols pour la prévision de crues : comparaison d'un modèle pluie-débit conceptuel et d'un modèle intégrant une interface sol-végétation-atmosphère. Revue des sciences de l'eau / Journal of Water Science, 16(2), 173-197. https://doi.org/10.7202/705503ar

\section{Résumé de l'article}

Le but de cet article est de présenter une méthodologie de mise à jour des paramètres de modèles pluie-débit en période de crue. Elle a été mise au point afin d'améliorer un des aspects de la gestion des réservoirs dans un contexte opérationnel de protection contre les crues: la réduction des incertitudes sur la prévision des débits. L'originalité de la méthode proposée réside dans le fait que l'on utilise non seulement une information sur les débits mais aussi une information sur l'humidité du sol. L'objectif de l'étude est d'évaluer l'intérêt de l'introduction de cette information supplémentaire. Pour cela, les données d'humidité du sol sont introduites au sein du modèle par l'intermédiaire d'une relation de passage établie entre l'humidité mesurée in situ et l'humidité calculée implicitement ou explicitement par les modèles. Cette méthodologie a été testée dans le cadre du projet européen AIMWATER sur quatre sous-bassins de la Seine en amont de Paris (France). Deux modèles pluie-débit sont utilisés dans cette étude, un modèle conceptuel semi-emprique et un modèle conceptuel couplé à un schéma de surface simulant une interface sol-végétation-atmosphère et permettant de calculer l'évolution de l'humidité du sol à différentes profondeurs. Cette approche comparative étudie l'intérêt d'un tel modèle couplé par rapport au modèle conceptuel semi-empirique sans représentation explicite des phénomènes se produisant à l'interface sol-végétation-atmosphère. 


\title{
Assimilation de données d'humidité des sols pour la prévision de crues : comparaison d'un modèle pluie-débit conceptuel et d'un modèle intégrant une interface sol-végétation-atmosphère
}

\author{
Assimilation of soil moisture into hydrological models \\ for flood forecasting: comparison of a conceptual \\ rainfall-runoff model and a model with an explicit \\ counterpart for soil moisture
}

\section{A. WEISSE*, L. OUDIN, C. LOUMAGNE}

Reçu le 30 octobre 2001, acceptation le 8 janvier $2003^{\text {** }}$

\section{SUMMARY}

\begin{abstract}
Improving the accuracy of rainfall-runoff models and in particular their performances in flood prediction is a key point of continental hydrology. Methods have been developed to improve flood prediction in hydrology based on a better compliance of the model with current observations prior to its use in forecasting mode. This operation has been termed updating in hydrology and assimilation in meteorology. The fundamental idea is that if model predictions diverge from observations at a given time, there is little chance that future estimations will approach correct values. The improvement then comes from a correction of the trajectory of the model based on observations during the period preceding the day when a prediction into the immediate or long-term future is desired. This can be dealt with by a correction of model parameters, which is usually called "parameter updating".
\end{abstract}

The inability of rainfall-runoff models to produce correct streamflow values generally translates into parameter uncertainty. Parameter calibration is the means used by a model structure to adjust to a given set of data. Therefore, a parameter updating methodology seems to be a natural way to amend errors in streamflow values. In this paper, a specific methodology of parameter updating is presented. The main feature of this method is that it does not carry out updating by reference only to recent streamflow observations, as classic procedures do, but also to soil moisture measurements, which can be retrieved daily from TDR (Time Domain Reflectometry) probes. Indeed, it

Cemagref, Parc de Tourvoie BP44, 92163 Antony Cedex France, tél. (33) 0140966053.

* Adresse actuelle : DIREN Lorraine, Service Hydrologie et Annonce de Crues - Cellule Études, 19, av. Foch, BP 60223, 57005 Metz Cedex 1, France.

* Correspondance. E-mail : anne.weisse@lorraine.environnement.gouv.fr

** Les commentaires seront reçus jusqu'au 30 décembre 2003. 
appears that the integration of soil moisture data allows better control of the evolution of the model and improves its performances, in particular in terms of forecasting.

The aim of the research was to assess the usefulness of this additional soil moisture information. To this end, an approach has been suggested that gradually introduces additional information thanks to a constraint relationship between observed and modelled soil moisture. In fact, soil moisture can be calculated implicitly or explicitly by the model when extracting step-by-step the values of the model's store contents. This methodology was put forward for use in the European AIMWATER project on four catchments within the Seine River basin upstream of Paris (France).

The other issue addressed in this paper was whether or not it is necessary to use a model that simulates explicitly the evolution of soil moisture at different depths. One can argue that if the model employed does not feature a store that can be identified closely to the observed soil moisture, there would be no possibility of benefiting from such measurements. On the other hand, it can be argued that if soil moisture is a model output, all the information drawn from soil moisture observations will be directed at improving this specific output at the expense of improving streamflow values. To answer this issue, two models were tested. The first model, GR4j, has no explicit counterpart for soil moisture measurements. The second one, GRHum, has been especially developed to introduce a two-layer soil reservoir that simulates the surface and sub-surface soil moisture.

Since the aim of the present research was to analyse different ways of accounting for soil moisture, and to identify the one that offers the best prospects, several tests were carried out, using different relationships between observed and modelled soil moisture. Indeed, TDR probes give point measurements of soil moisture at several depths and several store contents can be taken into account in a constraint relationship.

First, for both GR4j and GRHum models, tests showed that performances for flood forecasting are significantly improved when assimilating in situ measurements of soil moisture at a daily time-step, especially for the basins where poor simulations are obtained. It is also noteworthy that performances are very dependent on the items taken into account in a constraint relationship.

Secondly, the GRHum model did not appear to be more efficient than the GR4j model when assimilating both streamflow and soil moisture data. However, the GRHum model gave the best results when assimilating only streamflow data, and superficial soil moisture seemed to fit the GRHum better than the GR4j model.

Finally, although the tests required perfect foreknowledge of rainfall, the results of the research are encouraging from an operational point of view. Another interesting perspective is provided by the Earth Observation data. Indeed, previous studies have shown that soil moisture can be derived from EO data using, for example, microwave spaceborne Synthetic Aperture Radar (SAR) images (QUESNEY et al., 2000). This type of catchment-scale data could be more relevant than a local measure given by TDR probes (PAUWELS et al., 2002).

Key-words : assimilation, soil moisture, flood forecasting.

\section{RÉSUMÉ}

Le but de cet article est de présenter une méthodologie de mise à jour des paramètres de modèles pluie-débit en période de crue. Elle a été mise au point afin d'améliorer un des aspects de la gestion des réservoirs dans un contexte opérationnel de protection contre les crues: la réduction des incerti- 
tudes sur la prévision des débits. L'originalité de la méthode proposée réside dans le fait que l'on utilise non seulement une information sur les débits mais aussi une information sur l'humidité du sol. L'objectif de l'étude est d'évaluer l'intérêt de l'introduction de cette information supplémentaire. Pour cela, les données d'humidité du sol sont introduites au sein du modèle par l'intermédiaire d'une relation de passage établie entre l'humidité mesurée in situ et l'humidité calculée implicitement ou explicitement par les modèles. Cette méthodologie a été testée dans le cadre du projet européen AIMWATER sur quatre sous-bassins de la Seine en amont de Paris (France). Deux modèles pluie-débit sont utilisés dans cette étude, un modèle conceptuel semiemprique et un modèle conceptuel couplé à un schéma de surface simulant une interface sol-végétation-atmosphère et permettant de calculer l'évolution de l'humidité du sol à différentes profondeurs. Cette approche comparative étudie l'intérêt d'un tel modèle couplé par rapport au modèle conceptuel semi-empirique sans représentation explicite des phénomènes se produisant à l'interface sol-végétation-atmosphère.

Mots clés : assimilation, humidité du sol, prévision des crues.

\section{1 -INTRODUCTION}

Pour la gestion en temps réel des réservoirs et plus particulièrement dans un contexte de protection de la population contre les crues il faut pouvoir disposer d'outils opérationnels et fiables. II s'agit d'estimer à l'avance l'ampleur du phénomène pour pouvoir prendre les dispositions nécessaires afin d'atténuer les dégâts éventuels. Le gestionnaire doit pouvoir prévoir quelle va être l'évolution du débit de la rivière dans un futur proche afin d'optimiser la vidange de ses réservoirs. Une amélioration de la prévision des débits amène donc à une amélioration des opérations de gestion. En général, le problème nécessite au préalable des prévisions météorologiques et par conséquent, les résultats sont souvent donnés sous forme conditionnelle en fonction des pluies à venir. Souvent, le gestionnaire aura recours aux modèles pluie-débit pour prévoir au plus tôt les débits de crue. Ces méthodes permettent, en remontant à la pluie, de gagner un temps précieux par rapport aux modèles débit-débit basés sur des formules hydrauliques de propagation de l'onde de crue. Un des problèmes lié à l'utilisation de ces modèles est la connaissance des conditions initiales du bassin et notamment de son état hydrique. Les méthodes présentées dans cet article permettent de s'affranchir de cette limite.

Mais quels que soient les modèles utilisés, il existe de nombreuses sources d'erreurs qui sont susceptibles d'affecter la prévision résultante d'un événement particulier en dehors de la performance moyenne du modèle. Parmi elles figurent naturellement les différentes perturbations sur les données d'entrée, mais aussi l'adéquation du modèle de prévision à chaque événement de crue particulier. De nombreuses méthodes de mise à jour ont été développées pour améliorer la prévision des crues en modélisation hydrologique, allant d'une simple correction d'erreur à l'utilisation d'un réseau de neurones (ZEALAND et al., 1999). Parmi ces méthodes, la mise à jour par assimilation, définie comme étant l'utilisation simultanée de l'information contenue dans un modèle et dans un 
ensemble de données externes, a suscité de nombreux travaux non seulement en hydrologie mais aussi en océanographie, climatologie, météorologie..., domaines dans lesquels cette méthode est considérée comme présentant un grand potentiel d'amélioration des connaissances sur notre environnement (DALEY, 1997). Le processus d'assimilation permet d'établir un lien entre l'information externe, la variable observée, et les variables internes calculées par le modèle afin de corriger la divergence du modèle au cours de la prévision. La prévision se fait en dehors de la période de calage, une fois les paramètres optimisés. Si l'on fait confiance entièrement à la valeur prise par la variable observée, celle-ci peut venir directement remplacer les variables internes calculées par le modèle au cours de la prévision, c'est ce qu'on appelle le mode forcé. Si l'on ne fait pas entièrement confiance ni à la variable observée ni aux variables calculées par le modèle, alors on établit une relation pondérant la confiance accordée à chacune des informations.

Dans le domaine de la prévision de crues, ce sont essentiellement des méthodes d'assimilation de débits qui ont été développées. L'originalité de la démarche présentée ici est d'utiliser l'état hydrique des sols pour remettre à jour le système prévu. En effet, cette variable est une variable clé en hydrologie : elle affecte directement l'évaporation réelle, la répartition de la pluie entre infiltration et ruissellement et la percolation vers les couches de sol profondes. L'intérêt de prendre en compte une telle information dans les modèles hydrologiques a été étudié par plusieurs équipes de recherche (ENGMAN, 1990 ; LOUMAGNE et al., 1991 ; PIETRONIRO et al., 1993). Ces travaux ont montré l'intérêt de cette variable pour simuler les débits à l'échelle des bassins versants. C'est pourquoi, en vue d'améliorer les méthodes de prévision existantes, une nouvelle méthode a été proposée couplant à l'assimilation des débits une information supplémentaire : l'état hydrique du sol à travers des mesures d'humidité, afin d'étudier son influence sur la qualité des prévisions des crues (QUESNEY, 1999 ; WEISSE et al., 2001a ; WEISSE et al., 2001b ; OUDIN et al., 2001). Dans cette méthode, l'information externe est donc double : les débits et l'état hydrique du sol. Ces informations sont utilisées pour la mise à jour des paramètres du modèle de manière à corriger le système modélisé à partir d'observations sur les jours précédant le jour de la prévision, pour en limiter la divergence. L'incapacité du modèle à reproduire correctement les débits se traduit généralement par une incertitude sur les paramètres. Le calage des paramètres est donc un des moyens utilisé par le modèle pour s'adapter à un jeu d'observations donné. La remise à jour des paramètres sur une courte période précédant la prévision semble donc être une approche naturelle pour corriger l'erreur récente sur le débit calculé.

Le but de cet article est de montrer les résultats obtenus par l'assimilation des débits et des humidités du sol sur plusieurs bassins versants étudiés dans le cadre du projet européen AIMWATER (Analysis, Investigation and Monitoring of WATER resources, for the management of multi-purpose reservoirs), présenté dans LOUMAGNE et al., 2001. En particulier, deux modèles pluie-débit, GR4j (EDIJATNO et al., 1999 ; PERRIN, 2000) et GRHum (LOUMAGNE et al., 1996) ont été couplés à la méthode d'assimilation. Ce sont tous deux des modèles conceptuels globaux qui ont montré des performances équivalentes à celles obtenues avec d'autres modèles plus complexes trouvés dans la littérature (PERRIN et al., 2001). GR4j est un modèle semi-empirique à deux réservoirs sans représentation des échanges à l'interface sol-végétation-atmosphère alors que le modèle GRHum 
intègre un schéma de surface issu du modèle de DEARDORFF (1978) simulant ces échanges et qui calcule l'évolution des humidités superficielles et profondes du bassin versant. II a été développé afin de permettre une assimilation plus efficace des états hydriques superficiels obtenus à partir de l'observation spatiale. Nous évaluerons dans cet article l'intérêt respectif de chacun des modèles vis à vis de l'assimilation de données d'humidité des sols mesurées in situ.

\section{2 - MÉTHODE D'ASSIMILATION DE DONNÉES D'HUMIDITÉ DES SOLS POUR LA PRÉVISION DE CRUES}

\subsection{Méthodologies de mise à jour}

Malgré les améliorations faites en modélisation au cours de ces dernières décennies les modèles hydrologiques montrent toujours des limites dans leur capacité à prévoir les débits : les incertitudes sur les valeurs prévues demeurent encore trop élevées pour être utilisées dans un contexte opérationnel de prévision de crues. Les modèles utilisés dans ce contexte ne permettent pas d'évaluer correctement les débits prévus: il y a généralement un écart important entre les débits calculés par le modèle et les observations. Or si le modèle s'éloigne trop des observations à un moment donné, les prévisions auront peu de chance d'être fiables. L'amélioration des performances des modèles en prévision nécessite donc en premier lieu une correction de la trajectoire du modèle pour en limiter sa divergence. Quatre types de méthodologies de mise à jour ont été recensées en hydrologie (REFSGAARD, 1997, IDE et al., 1997). Elles diffèrent dans l'interprétation des origines des écarts entre les débits observés et simulés. La première suppose que le désaccord est dû aux erreurs faites sur l'estimation des données d'entrée du modèle (pluie, évapotranspiration potentielle, initialisation des réservoirs...). Dans cette approche, seules ces données sont corrigées afin d'améliorer les simulations du modèle. Cette méthodologie est appelée mise à jour des entrées (input updating). La deuxième méthodologie propose de modifier, durant les périodes à problèmes, les variables d'état du système afin que les simulations se rapprochent des observations (state updating). La troisième consiste à modifier les paramètres du modèle (parameter updating) au cours de la prévision. Les paramètres du modèle ayant été calés par optimisation sur une longue période d'observation, ils ne sont pas toujours bien adaptés lorsqu'il s'agit de prévoir des évènements particuliers tels que les crues. Une manière naturelle de réajuster le modèle au cours de la prévision est de modifier ces paramètres sur une courte période précédant le délai de prévision pour réduire la différence entre le débit observé et simulé le jour de la prévision. L'avantage de cette méthode est de ne pas modifier les quantités d'eau prises en compte et de conserver le bilan hydrique. Enfin, la quatrième méthodologie suppose que le désaccord est dû aux erreurs du modèle et aux erreurs sur les mesures (error correction).

Parmi ces méthodes, deux d'entres elles (state \& parameter updating) sont plus particulièrement adaptées aux modèles non linéaires tels que les modèles conceptuels utilisés. Associés à ces méthodes deux types d'algorithmes ont été développés dans le cadre d'AIMWATER pour réajuster les modèles pluie-débit à 
partir des observations : l'assimilation variationnelle, qui réajuste le modèle sur une période précédant la prévision et l'approche séquentielle à partir d'un filtre de Kalman étendu (AUBERT et al., 2001) qui réajuste le modèle seulement lorsqu'une observation est disponible, les nouvelles estimations fournissant alors les nouvelles conditions initiales du modèle. Nous ne présenterons dans ce papier que la méthode et les résultats issus de la méthode variationnelle, la méthode séquentielle n'ayant pas encore été validée sur l'ensemble des bassins.

\subsection{Méthode d'assimilation variationnelle des débits et des humidités de sol}

Le but de la démarche est d'étudier l'amélioration apportée par la mesure de l'humidité du sol dans une méthode de mise à jour des paramètres basée sur l'assimilation variationnelle des débits (YANG, 1993 ; YANG et MICHEL, 2000). La méthodologie développée a déjà été exposée en détail (WEISSE et al., $2001 b)$ et seules les grandes lignes de l'assimilation couplée débits et humidités sont rappelées ici.

Cette méthodologie se différencie de l'assimilation variationnelle des débits par l'ajout d'une contrainte supplémentaire utilisant l'information sur l'état hydrique du sol. II existe de nombreuses solutions permettant de réduire l'erreur récente sur le débit : prise en compte des incertitudes sur les pluies, sur l'évapotranspiration potentielle.... L'hypothèse faite ici est que l'humidité du sol, variable de contrôle des débits est l'information qui peut être exploitée au mieux pour modifier les paramètres du modèle dans la procédure de mise à jour. L'humidité des sols peut être utilisée pour améliorer la simulation des débits de façon plus vraisemblable, c'est-à-dire en rendant mieux compte des observations récentes d'humidité. Cette information peut aider à choisir une solution qui serait plus proche de la réalité tant au niveau des débits que des humidités et qui serait donc plus performante que la méthode d'assimilation des débits seuls.

La première étape de cette méthode couplée, effectuée avant toute opération de mise à jour, est basée sur une analyse préliminaire des erreurs des débits simulés $Q_{\text {sim }}$ par rapport aux débits observés $Q_{o b s}$ en période de crue (LAMB, 1999). Ces périodes étant définies comme étant des périodes où le débit est supérieur à 4 fois le débit moyen interannuel:

$$
Q_{o b s}=\alpha+\beta Q_{\text {sim }}+\varepsilon
$$

où $Q_{\text {obs }}$ est le débit observé, $Q_{\text {sim }}$ est le débit calculé par le modèle, $\alpha, \beta$ les paramètres de la régression linéaire et $\varepsilon$ le terme d'erreur.

En effet, il existe un biais systématique entre le débit observé et calculé plus particulièrement en période de crues ( $\alpha$ est significativement différent de zéro et $\beta$ significativement différent de 1). II est donc important de corriger ce biais préalablement à toute mise à jour afin de rendre l'erreur indépendante du débit calculé et améliorer sa qualité statistique avant sa mise à jour. C'est donc le débit corrigé déduit de cette équation qui est utilisé par la suite dans la méthodologie.

La même analyse est faite pour les humidités. En effet, QUESNEY (1999) a montré qu'il existe des relations entre les états internes du modèle GRHum et les humidités mesurées in situ, pour le bassin de l'Orgeval. Ces résultats ont pu être étendus à plusieurs bassins versants et au modèle GR4j. De manière générale, il est donc possible de relier les états internes du modèle (états des réservoirs) aux humidités observées $\mathrm{H}_{\mathrm{o}}$, par régression linéaire en période de crue. 


$$
\mathrm{H}_{0}=\gamma+\delta \mathrm{Hc}+\eta
$$

où $\mathrm{H}_{\mathrm{o}}$ est l'humidité journalière mesurée in situ, $\mathrm{H}_{\mathrm{c}}$ est le contenu en eau des réservoirs calculé par le modèle, $\gamma, \delta$ les paramètres de la régression linéaire et $\eta$ le terme d'erreur. Les relations établies sont spécifiques à chaque bassin étudié.

La deuxième étape de cette méthode est la procédure de mise à jour appliquée lorsqu'une prévision est faite. Le principe de cette procédure consiste à mettre à jour les valeurs des paramètres du modèle en utilisant les observations de débit et d'humidité disponibles sur une période antérieure au jour de prévision. On suppose que l'on peut pallier les écarts récents entre modèle et observation en modifiant provisoirement le jeu de paramètres du modèle, pour mieux s'adapter à la situation en cours. Ces modifications sont effectuées sur une période de rétroaction longue ( 60 jours) déterminée par optimisation de la procédure, de manière à ce qu'elles aient un impact réel sur le débit simulé le jour de la prévision. Lorsque les modifications des paramètres permettent d'obtenir une erreur minimale sur le dernier débit observé $\mathrm{j}$, on peut réaliser une prévision aux jours $j+L(L=$ délai de prévision compris entre 1 et 2 jours dans notre cas). A la différence du calage où tous les débits sont connus, il s'agit de faire un pari sur la stratégie de correction à apporter sans connaître le débit réel au jour de la prévision. Les nouvelles simulations du modèle doivent respecter les deux conditions suivantes:

II s'agit de réduire tout d'abord, la différence entre les débits observés $Q_{o b s}$ et corrigés $Q_{\text {sim }}$ par l'équ. (1) sur les derniers débits observés $(j-k, k=0$ à 5 ) en ajustant les paramètres du modèle de manière à mieux prévoir les débits à venir. Pour chaque prévision, le jeu de paramètres déterminé a priori sur la période de calage fournit les conditions initiales de l'optimisation. Le critère initial (Crit) calculé sans mise à jour des paramètres doit être réduit au cours de la mise à jour :

$$
\text { Crit }=\sum_{k=0}^{K} p\left|Q_{o b s, j-k}-Q_{p r e ́ v u, j-k}\right|
$$

où $Q_{\text {prévu }}$ est le débit mis à jour après modification des paramètres et $p(k)$ un facteur de pondération croissant en fonction de $k$, traduisant l'hypothèse que plus une erreur est proche du jour de prévision plus elle risque d'occasionner des erreurs importantes en prévision.

II s'agit ensuite, de respecter une contrainte supplémentaire sur les humidités observées et modélisées par le modèle pluie-débit le jour de la prévision :

$$
|H o(j)-H c(j)|<\frac{\sigma}{k}
$$

où Ho représente les humidités mesurées in situ, $\mathrm{Hc}$ le contenu en eau des réservoirs calculé par le modèle (réservoir superficiel, profond ou de routage), $\sigma$ l'écart type de Hc sur la période de rétroaction et $k$ un paramètre qui impose la sévérité de la contrainte (plus k est grand, plus elle est prise en compte). La valeur de $\mathrm{k}$ donne une mesure de l'intérêt de la prise en compte de l'humidité. Cette valeur k est liée à l'humidité observée, elle est donc spécifique à chaque bassin versant.

Tant que la condition (4) est vérifiée sur la période de rétroaction, l'erreur sur les humidités peut varier sans dépasser le seuil $\frac{\sigma}{k}$. Lorsque cette condition n'est pas vérifiée, le nouveau jeu de paramètres est rejeté. 
La procédure d'assimilation des débits et des humidités s'appuie donc sur deux contraintes :

(i) le nouveau jeu de paramètres doit réduire pendant la période de rétroaction les erreurs sur les derniers débits observés (3)

(ii) ce jeu doit satisfaire la condition (4) de contrainte sur les humidités, sinon il est rejeté.

\subsection{Critère de qualité des prévisions de crue}

Le critère de qualité de la prévision utilisé ici est le critère de persistance proposé par KITANDINIS et BRAS, (1980), c'est l'erreur quadratique des débits prévus $Q_{\text {prévu }}$ par rapport aux débits observés $Q_{\text {obs. }}$. La différence avec la formulation initiale est la durée du délai de prévision $L$ qui peut être ici supérieure à 1 jour. II est évalué sur les périodes où des prévisions sont faites, c'est-àdire, pour chaque crue observée, il est calculé sur toute la période où les débits sont supérieurs à 4 fois le débit moyen interannuel.

$$
\text { Pers }=100\left[1-\frac{\sum_{j}^{n}\left(Q_{o b s}(j+L)-Q_{p r e ́ v u}(j+L)\right)^{2}}{\sum_{j}^{n}\left(Q_{o b s}(j+L)-Q_{o b s}(j)\right)^{2}}\right]
$$

où $n$ est le nombre de débits faisant l'objet d'une prévision et $L$ le délai de prévision de 1 ou 2 jours en fonction de la taille du bassin. Ce critère compare les performances de la procédure de mise à jour des paramètres à celles qui seraient obtenues par une prévision «persistante " qui suppose que le débit du jour $\mathrm{j}+\mathrm{L}$ est égal au débit du jour j. Ceci permet de focaliser le critère de qualité sur la détection des changements de régime. Ce critère est compris entre [- $\infty$ et $100 \%$ ] lorsque le critère est égal à $0 \%$ la méthode est équivalente à la méthode «persistante », une valeur négative indique que la méthode est moins performante et une valeur positive indique que la mise à jour est plus performante que la prévision " persistante ».

MODÈLES HYDROLOGIQUES UTILISÉS : GR4j ET GRHUM

II existe un très grand nombre de modèles en hydrologie, qui permettent de décrire le fonctionnement du bassin versant par une représentation plus ou moins simplifiée de la réalité. AMBROISE (1999) a réalisé un inventaire des modèles en les différenciant par leurs concepts majeurs utilisés et selon la description de leurs relations internes (modèle empirique, conceptuel ou physique), la nature de leurs variables et paramètres (modèle déterministe ou stochastique), l'unité spatiale modélisée (modèle global ou distribué) ou la nature des processus décrits (modèle cinétique ou dynamique).

Les modèles que nous allons utiliser ici s'apparentent selon la définition d'Ambroise à des modèles conceptuels globaux, les processus physiques sont donc simplifiés et non spatialisés. Toujours selon cette définition, GR4j, est un 
modèle conceptuel semi-empirique sans lien explicite avec les processus de surface, alors que le modèle GRHum utilise un schéma de surface simulant une interface sol-végétation-atmosphère, permettant de modéliser à la fois l'humidité d'une couche de sol superficielle et l'humidité d'une couche de sol globale.

Le modèle GR4j-modèle du Génie Rural à 4 paramètres Journalier- (EDIJATNO et al., 1999 ; PERRIN, 2000) est un modèle journalier qui a été développé pour être utilisé sur des bassins versants non-jaugés. La fonction de transfert (ou routage) est gérée par 3 paramètres ( $B$ : capacité maximale du réservoir de transfert et $C$ : durée de base des hydrogrammes unitaires et $D$ : échanges souterrains). La fonction de production est gérée par 1 autre paramètre ( $A$ : capacité maximale du réservoir de production (ou sol)). La version retenue ici (figure 1) est celle mise au point par PERRIN (2000). Elle inclut notamment un terme supplémentaire de percolation Perc du réservoir de production.

\begin{tabular}{|l|}
\hline Paramètres : \\
A : Capacité du réservoir de production \\
B : Capacité du réservoir de routage \\
C : Durée de base des hydrogrammes \\
D : Paramètre des échanges souterrains \\
\hline
\end{tabular}

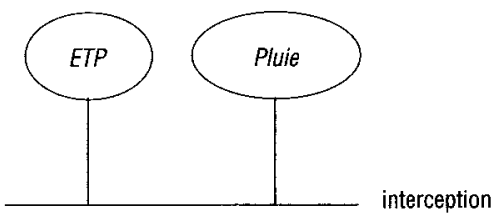

Notations :
Pn: Pluie nette
$P s:$ Pluie alimentant le réservoir
de production
Etp: Evapotranspiration potentielle
En: Evapotranspiration nette
Es : Evapotranspiration du réservoir
de production
$S:$ Niveau du réservoir
de production
$\boldsymbol{R}:$ Niveau du réservoir de routage
Perc : Percolation du réservoir
de production
$P r:$ Production totale
UHIet $2:$ Hydrogrammes unitaires
ECH: échanges souterrains

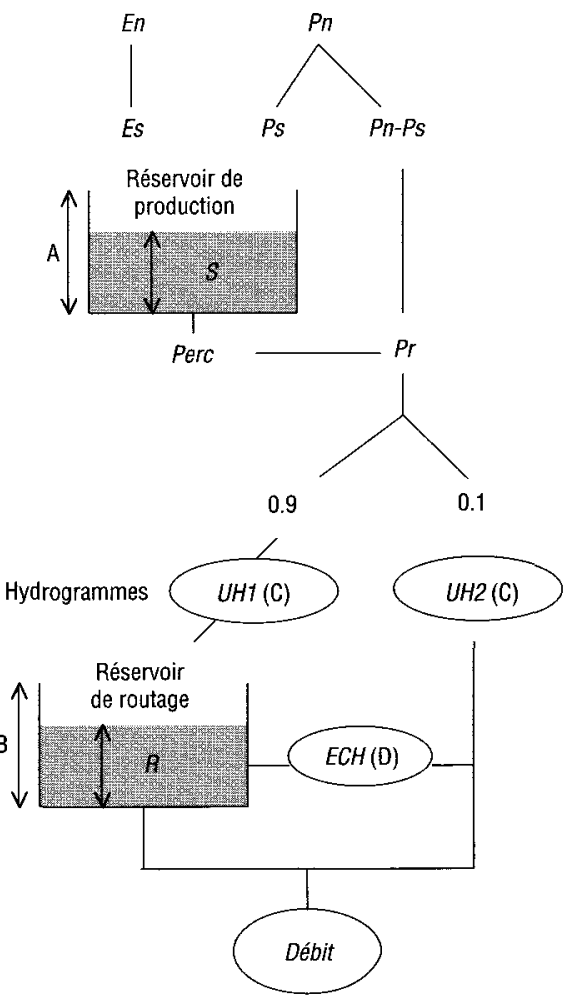

Figure 1 Structure du modèle GR4j.

Schematic diagram of the GR4j model. 
Le modèle GRHum-modèle du Génie Rural avec simulation de l'HUMidité(LOUMAGNE et al., 1996) est un aussi un modèle journalier conceptuel global, développé pour tester l'apport d'une prise en compte des indices hydriques. II a été développé à partir du modèle GR4j. La fonction de transfert est la même, seule la fonction de production diffère : en particulier, le réservoir sol (ou production) a été modifié de manière à introduire une couche superficielle de sol comprise dans la couche globale, afin d'obtenir des simulations d'humidités superficielles comparables aux indices hydriques déduits de la télédétection radar. La version utilisée dans cette étude (figure 2) comporte seulement 6 paramètres à caler ( 3 gérant la fonction de production Wgmax, Wsmax contenu en eau maximal des couches globales et superficielles et $\mathrm{K}$ taux de couverture végétale, 3 gérant la fonction de transfert les mêmes que dans le modèle GR4j). Ces paramètres sont optimisés pendant la période de calage en maximisant le critère de Nash. Cette période est différente de la période de validation où sont effectuées les prévisions. Ce sont les états internes calculés par le modèle $\mathrm{Wg}$, Ws et $\mathrm{R}$ qui sont utilisés pour contraindre le système pendant la mise à jour du modèle. Cette version simplifiée (figure 2) permet une application plus aisée de GRHum, sans collecte de données sur l'occupation des sols chaque année et pour chaque bassin versant. Ce qui se traduit par :

(i) La production de la couche globale PRg est définie comme une fonction seuil :

$$
\text { si } W g>W g \text { max, } P R g=W g-W g_{\text {max }} \text {, sinon } P R g=0
$$

(ii) Le taux de couverture végétale évaporante $\sigma_{f}$, qui représente le taux de végétation verte apte à transpirer, est modélisé comme une fonction de l'évapotranspiration potentielle (ETP de Penman):

$$
\sigma_{f}=K \frac{E T P}{E T P_{\max }}
$$

où $\mathrm{K}$ est un paramètre positif optimisé pendant la période de calage, compris entre 0 et 1 , et ETP ${ }_{\max }$, l'évapotranspiration potentielle maximale enregistrée.

Ce taux de couverture végétale est en particulier utilisé pour modéliser les évaporations réelles de la couche globale $E_{g}$ et de la couche de surface $E_{s}$ (cf. LOUMAGNE et al., 1996). L'évaporation de la couche globale $E_{g}$ se décompose entre l'évaporation du sol nu, $E_{\text {sol, }}$ calculée en se basant sur le concept d'évaporation limite (WETZEL et al., 1987) et la transpiration des plantes $E_{\text {veg }}$ qui se déduit de l'évaporation potentielle suivant une relation empirique validée dans le cadre de l'expérience Hapex-Mobilhy (OTTLE et VIDAL-MADJAR, 1994). L'évaporation de la couche de surface, $\mathrm{E}_{\mathrm{s}}$, se décompose entre l'évaporation du sol nu, une partie assez faible $(20 \%)$ de la transpiration des plantes estimée d'après la répartition racinaire verticale, et le contenu en eau de la couche superficielle, Ws qui est un facteur limitant pour l'évaporation.

$$
\begin{gathered}
\mathrm{E}_{\mathrm{g}}=\left(1-\sigma_{\mathrm{f}}\right) \mathrm{E}_{\text {sol }}+\sigma_{\mathrm{f}} E_{v e g} \\
\mathrm{E}_{\mathrm{s}}=\left(1-\sigma_{\mathrm{f}}\right) \mathrm{E}_{\text {sol }}+0.2 \sigma_{\mathrm{f}} \mathrm{E}_{\text {veg }} \frac{W_{s}}{W_{s \max }}
\end{gathered}
$$




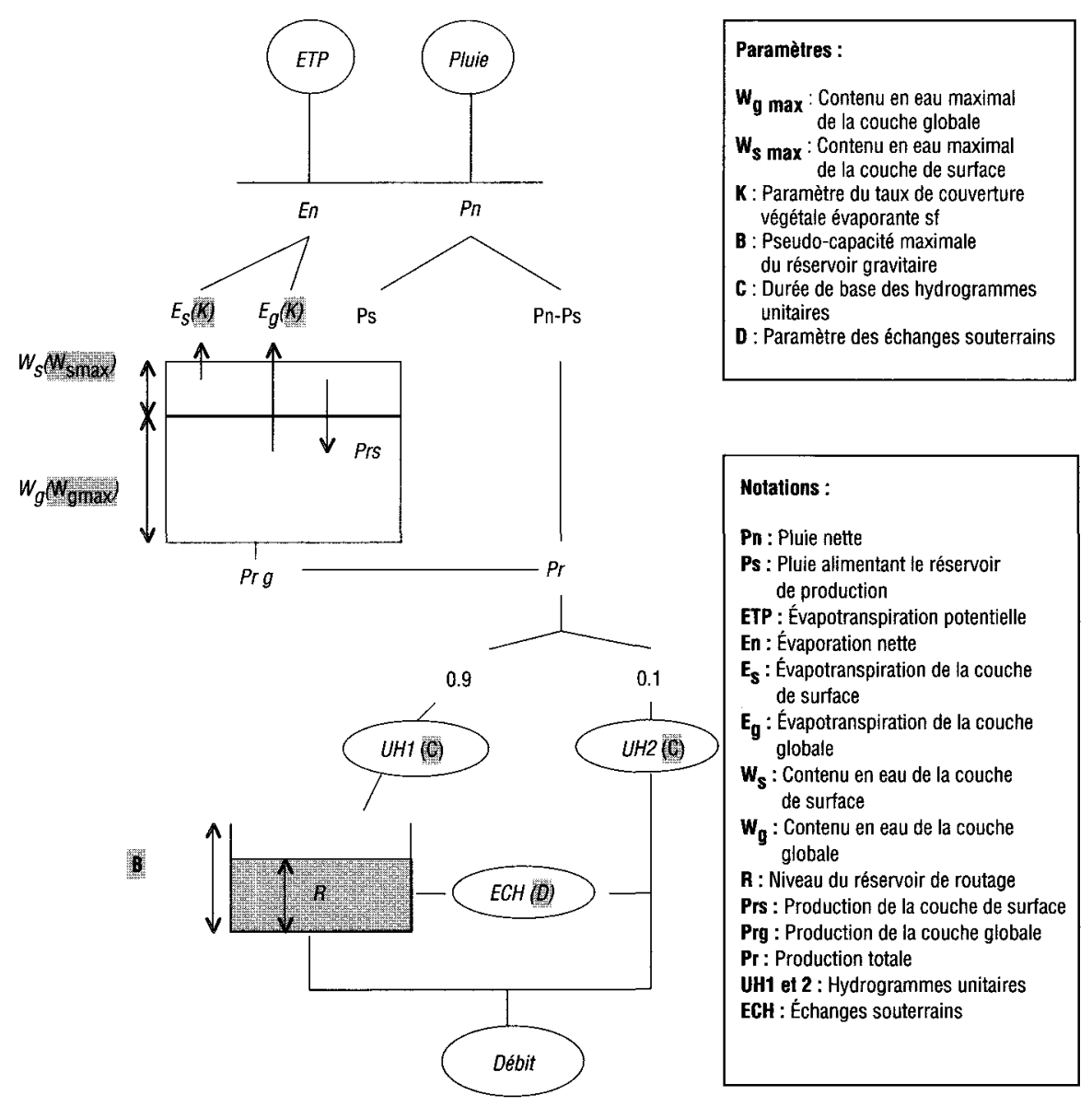

Figure 2 Structure du modèle GRHum.

Schematic diagram of the GRHum model.

\section{4 - BASSINS VERSANTS ÉTUDIÉS}

\subsection{Données hydro-météorologiques et données d'humidité des sols pour les bassins versants}

Les bassins versants choisis pour mettre au point la méthodologie d'assimilation de données d'humidité sont des sous bassins versants de la Seine (figure 3) : le Serein (1120 km²), affluent de l'Yonne, le Grand Morin (1070 km²), l'Orgeval (104 km²), qui est un sous bassin versant du Grand Morin, et le Petit 
Morin $\left(605 \mathrm{~km}^{2}\right)$. Le Grand Morin et le Petit Morin sont des affluents de la Marne. Tous ces bassins sont principalement agricoles, avec un relief peu marqué.

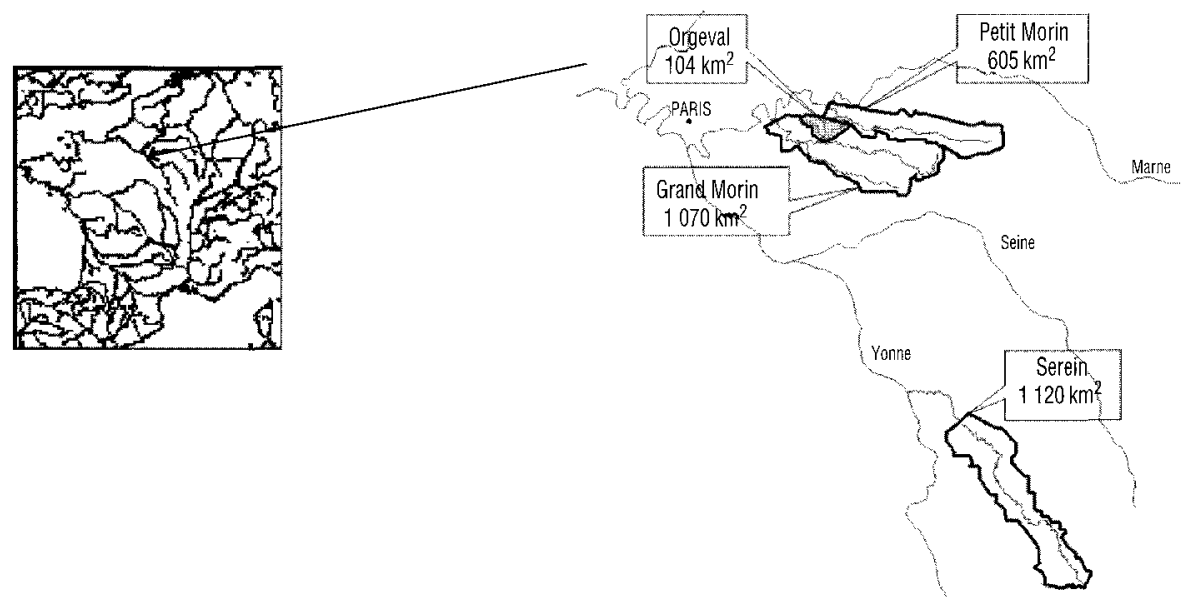

Figure 3 Carte de localisation des bassins versants étudiés.

Map showing the four studied watersheds.

Une base de données hydro-météorologique a été rassemblée pour chacun des bassins (tableau 1 et 2). Les modèles sont utilisés au pas de temps journalier et les données sont donc intégrées sur ce pas de temps. Les débits journaliers sont extraits de la banque de données HYDRO du Ministère de l'Environnement, les pluies journalières et les évapotranspirations potentielles décadaires datées (Penman) sont extraites de la base de données COLCHIQUE de Météo France. Enfin, l'Orgeval est un bassin expérimental géré par le Cemagref. Pour chaque bassin versant, les pluviomètres ont été choisis de manière à ce que la couverture spatiale du bassin soit la plus homogène possible ce qui permet d'évaluer chaque jour une moyenne par bassin.

Les données d'humidité utilisées dans cette recherche sont celles mesurées toutes les $12 \mathrm{~h}$ et intégrées au pas de temps journalier, par des sondes TDR placées horizontalement à différentes profondeurs du sol (tous les $10 \mathrm{~cm}$, entre $5 \mathrm{~cm}$ et $105 \mathrm{~cm}$ ) en deux sites différents : l'un sur le Serein et l'autre sur l'Orgeval. L'hypothèse faite est que l'évolution de l'état hydrique du sol mesurée par les sondes TDR est un bon indicateur de l'évolution de l'état hydrique des bassins versants. En effet les mesures gravimétriques effectuées (une vingtaine de campagnes par an) par prélèvement (une vingtaine d'échantillons par parcelle) sur une dizaine de parcelles témoins par bassin, montrent que l'évolution moyenne de ces prélèvements est en bon accord avec le suivi enregistré par les sondes TDR tout au long de l'année. II serait cependant faux d'affirmer que ces mesures ponctuelles sont représentatives de l'état hydrique du bassin versant compte tenu de la forte variabilité spatiale de cette grandeur. Les tech- 
niques de mesure par télédétection sont un bon moyen pour pallier à ce problème: îs fournissent des outils permettant de caractériser instantanément des zones étendues. La télédétection radar a montré son intérêt pour le suivi de l'humidité superficielle au cours du cycle hydrologique en donnant une information spatialisée, représentative à l'échelle du bassin versant. Une méthodologie a été mise au point pour déterminer des indices hydriques superficiels à l'échelle de bassins versants à partir de données du radar à synthèse d'ouverture (SAR) du satellite européen ERS (QUESNEY et al., 2000). L'inconvénient de ces données est leur faible répétitivité temporelle actuelle, incompatible avec la prévision des crues: deux mesures pour 35 jours seulement, c'est pour cette raison qu'elles n'ont pas été utilisées ici mais ont été étudiés dans le cadre du projet AIMWATER (OUDIN et al., 2001).

Tableau 1 Données hydro-météorologiques et données d'humidité des sols rassemblées pour les bassins versants.

Table 1 Hydro-meteorological and soil moisture data over the studied catchments.

\begin{tabular}{|c|c|c|c|c|}
\hline & $\begin{array}{l}\text { Débit à } \\
\text { l'exutoire }\end{array}$ & $\begin{array}{l}\text { Pluie moyenne } \\
\text { par bassin }\end{array}$ & $\begin{array}{l}\text { Evapotranspiration } \\
\text { (station météo) }\end{array}$ & $\begin{array}{l}\text { Humidité des sols } \\
\text { (mesures TDR } \\
\text { ponctuelles } \\
5-105 \mathrm{~cm} \text { ) }\end{array}$ \\
\hline $\begin{array}{l}\text { Pas de temps } \\
\text { des données }\end{array}$ & $\begin{array}{l}\text { Intégré } \\
\text { sur } 24 \mathrm{~h}\end{array}$ & $\begin{array}{l}\text { Intégrée } \\
\text { sur } 24 \mathrm{~h}\end{array}$ & $\begin{array}{c}\text { Donnée décadaire } \\
\text { datée }\end{array}$ & $\begin{array}{l}\text { Intégrée } \\
\text { sur 24h }\end{array}$ \\
\hline $\begin{array}{c}\text { Serein } \\
\left(1120 \mathrm{~km}^{2}\right)\end{array}$ & $\begin{array}{l}\text { Sep. 1955- } \\
\text { Fév. } 2001\end{array}$ & $\begin{array}{l}\text { Jan. } 1955- \\
\text { Fév. } 2001 \\
9 \text { pluviomètres }\end{array}$ & $\begin{array}{l}\text { Moyenne interannuelle } \\
\text { des données } \\
\text { décadaires datées }\end{array}$ & $\begin{array}{l}\text { Fév. 1999- } \\
\text { Jan. } 2001\end{array}$ \\
\hline $\begin{array}{c}\text { Orgeval } \\
\left(104 \mathrm{~km}^{2}\right)\end{array}$ & $\begin{array}{l}\text { Jan. 1963- } \\
\text { Fév } 2001\end{array}$ & $\begin{array}{l}\text { Jan. } 1963- \\
\text { Déc. } 2001 \\
5 \text { pluviomètres }\end{array}$ & & $\begin{array}{l}\text { Sept. 1997- } \\
\text { Jan. } 2001\end{array}$ \\
\hline $\begin{array}{l}\text { Grand Morin } \\
\left(1070 \mathrm{~km}^{2}\right)\end{array}$ & $\begin{array}{l}\text { Jan. 1994- } \\
\text { Jan. } 2001\end{array}$ & $\begin{array}{l}\text { Jan. 1994- } \\
\text { Fév. 2001 } \\
8 \text { pluviomètres }\end{array}$ & & $\begin{array}{l}\text { Sept. 1997- } \\
\text { Jan. } 2001\end{array}$ \\
\hline $\begin{array}{l}\text { Petit Morin } \\
\left(605 \mathrm{~km}^{2}\right)\end{array}$ & $\begin{array}{l}\text { Jan. 1970- } \\
\text { Jan. } 2001\end{array}$ & $\begin{array}{l}\text { Jan. } 1970- \\
\text { Fév. } 2001 \\
7 \text { pluviomètres }\end{array}$ & & $\begin{array}{l}\text { Sept. 1997- } \\
\text { Jan. } 2001\end{array}$ \\
\hline
\end{tabular}

Tableau 2 Périodes de calage utilisées pour les différents bassins versants.

Table 2 Calibration periods over the studied area.

\begin{tabular}{cccc}
\hline & $\begin{array}{c}\text { Période de mesures } \\
\text { hydro-pluviométriques }\end{array}$ & $\begin{array}{c}\text { Période de mesures } \\
\text { d'humidité (in situ) }\end{array}$ & Période de calage \\
\hline Serein & $1955-2001$ & $1999-2001$ & $1955-1997$ \\
Orgeval & $1963-2001$ & $1997-2001$ & $1963-1995$ \\
Grand Morin & $1994-2001$ & $1997-2001$ & $1994-1996$ \\
Petit Morin & $1970-2001$ & $1997-2001$ & $1970-1995$ \\
\hline
\end{tabular}




\subsection{Modélisation hydrologique des bassins versants étudiés}

Le critère de Nash a été retenu pour évaluer la qualité des modèles en calage (tableau 2) et en simulation sur les bassins versants :

$$
N_{a}=100\left(1-\frac{\sum_{j}\left(Q_{o b s, j}-Q_{s i m, j}\right)^{2}}{\sum_{j}\left(Q_{o b s, j}-\bar{Q}\right)^{2}}\right)
$$

où $Q_{o b s, j}$, est le débit observé le jour j, $Q_{\text {sim, }}$, le débit simulé par le modèle au jour j et $\bar{Q}$ la moyenne interannuelle des débits observés. Plus le critère de Nash se rapproche de 100 , plus les débits simulés sont proches des débits observés. Dans la procédure de calage des paramètres du modèle, ce critère doit être maximisé.

Le critère de Nash (équation 9 et tableau 3) est un critère qui met l'accent sur les périodes de crues. En effet, la sommation du carré des différences entre les débits observés et simulés conduit à privilégier les performances du modèle sur les périodes pour lesquelles ces différences sont les plus élevées, donc particulièrement en périodes de crues. Le tableau 3 montre également les critères de Nash évalués sur les racines carrées et les logarithmes des débits, ce qui permet d'évaluer la sensibilité du modèle aux débits moyens et aux étiages respectivement.

Tableau 3 Performances en calage des modèles GR4j et GRHum sur les bassins d'étude : critère de Nash (\%).

Table 3 Performances of GR4j and GRHum in calibration mode on the studied area: Nash criteria values.

\begin{tabular}{cccccccc}
\hline & \multicolumn{3}{c}{ GR4j } & & \multicolumn{3}{c}{ GRHum } \\
\cline { 2 - 3 } \cline { 7 - 8 } & Na (Q) & Na $(\sqrt{ } \mathbf{a})$ & Na $(\operatorname{In} \mathbf{Q})$ & & Na (a) & Na $(\sqrt{ } \mathbf{a})$ & Na $(\operatorname{In} \mathbf{Q})$ \\
\hline Serein & 88,2 & 88,7 & 81,7 & & 87,6 & 85,1 & 71,5 \\
Orgeval & 77,8 & 78,9 & 70,8 & & 78,3 & 77,4 & 68,9 \\
Grand Morin & 83,2 & 46,8 & $-112,5$ & & 86,6 & 61,7 & $-35,5$ \\
Petit Morin & 81,9 & 84,1 & 76,2 & & 77,8 & 78,8 & 69,5 \\
\hline
\end{tabular}

Le calage des paramètres pour les bassins versants étudiés a été fait sur des périodes de données hydrométriques et pluviométriques les plus longues possible, de manière à ce que le jeu de paramètres soit optimum pour le bassin, c'est-à-dire qu'il s'adapte au plus grand nombre de situations météorologiques possible rencontrées. Afin de tester l'assimilation des données d'humidité, les périodes de calage (tableau 2) s'arrêtent un an avant le début de la collecte des humidités in situ (fin 97 pour le Serein, fin 95 pour les autres bassins excepté le Grand Morin où la base de données est très réduite : la période de calage empiète d'un an sur celle des données d'humidité, afin d'allonger la période de calage et la rendre plus significative). Cette période d'un an correspond à la période de mise en route du modèle. 
Les performances des modèles en simulation des débits sur toute la période de calage sont présentées dans le tableau 3. De manière générale, les performances des modèles GR4j et GRHum sont élevées et équivalentes sur les différents bassins, sauf pour le Grand Morin, bassin pour lequel les modèles sont mis en défaut pour la simulation des étiages (critère de Nash négatif sur les logarithmes des débits). Ces valeurs négatives du critère de Nash peuvent s'expliquer par le fait que sur ce bassin la période de calage est très courte ( 3 ans) et il existe un problème de mesure de ces débits signalé dans la banque de données: la station de jaugeage est située très près de la confluence Marne-Grand Morin, les niveaux d'étiage sont donc très influencés par ceux de la Marne. Il est à noter aussi que les performances en simulation sont particulièrement élevées pour le bassin du Serein avec des critères de Nash supérieurs à $85 \%$.

\section{5 - RESULTATS DE LA METHODE D'ASSIMILATION}

L'objectif de l'étude étant de mesurer l'intérêt de l'introduction d'une information sur l'humidité du sol dans la méthode d'assimilation variationnelle, nous présenterons donc dans un premier temps les résultats obtenus avec assimilation des débits seuls, puis dans un deuxième temps les résultats obtenus avec assimilation supplémentaire des humidités. Les deux méthodes d'assimilation sont très proches, la seule différence dans le dernier cas étant l'ajout d'une contrainte supplémentaire sur les humidités et qui a été décrite à la section 2.2.

\subsection{Résultats de l'assimilation des débits}

La méthode initiale assimilant uniquement les débits a été testée sur les différents bassins versants pour les mêmes périodes de crues qui seront ensuite utilisées avec les mesures d'humidité (tableau 4). II s'agit d'évaluer dans un premier temps l'efficacité de l'utilisation seule des débits pour la mise à jour des paramètres. II faut préciser que, dans ce travail, nous ne ferons pas d'essais de prévision de crue avec des scénarii de pluies mais avec des pluies connues. Ceci nous permet de tester uniquement la méthode sans faire intervenir de biais engendré par les prévisions météorologiques.

Tableau 4 Période de test de la méthode et nombre de crues prises en compte.

Table 4 Test periods for the studied catchments and number of flood events.

\begin{tabular}{cccc}
\hline & $\begin{array}{c}\text { Période } \\
\text { de calage }\end{array}$ & Période de test & $\begin{array}{c}\text { Nombre de crues } \\
\text { de la période test }\end{array}$ \\
\hline Serein & $1955-1997$ & $1999-2001$ & 12 \\
Orgeval & $1963-1995$ & $1997-2001$ & 38 \\
Grand Morin & $1994-1996$ & $1998-2001$ & 29 \\
Petit Morin & $1970-1995$ & $1997-2001$ & 16 \\
\hline
\end{tabular}


Les résultats obtenus en prévision de crues et pluies connues (tableau 5) sont des résultats issus de la période test c'est-à-dire, des résultats en validation sans réoptimisation des paramètres. Au jour de la prévision $j+L$, il n'y a pas de remise à jour des paramètres, les résultats sont calculés avec le meilleur jeu de paramètres issu de la période rétroactive précédant la prévision. Ces résultats sont présentés pour les deux modèles hydrologiques GR4j et GRHum. La méthode d'assimilation des débits est comparée à une méthode de référence sans assimilation : c'est en fait les résultats d'une simulation simple avec pluies connues pour les mêmes périodes de crues. D'autre part, les délais de prévision ont été choisis par rapport au temps de réponse (paramètre $\mathrm{C}$ ) des modèles pour chaque bassin versant. Ces temps de réponse sont liés à la taille du bassin considéré. Ils vont jusqu'à 2 jours pour des bassins versants de taille conséquente (Serein, Grand Morin et Petit Morin) et 1 jour pour l'Orgeval.

Tableau 5 Comparaison en prévision de crues par simulation simple et par assimilation des débits pour les modèles GR4j et GRHum (persistance en \%).

Table 5 Comparison of the performances of flood forecasting in simulation mode and with streamflow assimilation using GR4j and GRHum models over the studied area (persistence criterion).

\begin{tabular}{|c|c|c|c|c|}
\hline & \multicolumn{2}{|c|}{$\begin{array}{c}\text { Sans mise à jour des paramètres } \\
\text { (simulation) }\end{array}$} & \multicolumn{2}{|c|}{$\begin{array}{l}\text { Méthode variationnelle } \\
\text { assimilation des débits }\end{array}$} \\
\hline & GR4j & GRHum & GR4j & GRHum \\
\hline Serein & $\begin{array}{c}61,0(\mathrm{~L}=1 \text { jour }) \\
86,1(\mathrm{~L}=2 \text { jours })\end{array}$ & $\begin{array}{l}58,7(\mathrm{~L}=1 \text { jour }) \\
85,3 \text { ( } L=2 \text { jours })\end{array}$ & $\begin{array}{c}80,9(\mathrm{~L}=1 \text { jour }) \\
\mathbf{8 5 , 5}(\mathrm{L}=2 \text { jours })\end{array}$ & $\begin{array}{c}83,0(\mathrm{~L}=1 \text { jour }) \\
87,5(\mathrm{~L}=2 \text { jours })\end{array}$ \\
\hline Orgeval & $69,4$ ( $L=1$ jour $)$ & $69,4(\mathrm{~L}=1$ jour $)$ & $\mathbf{6 0 , 5}(\mathrm{L}=1$ jour $)$ & $63,4(\mathrm{~L}=1$ jour $)$ \\
\hline Grand Morin & $\begin{array}{r}69,6(\mathrm{~L}=1 \text { jour }) \\
84,8(\mathrm{~L}=2 \text { jours })\end{array}$ & $\begin{array}{c}67,0(\mathrm{~L}=1 \text { jour }) \\
83,4(\mathrm{~L}=2 \text { jours })\end{array}$ & $\begin{array}{c}73,5(L=1 \text { jour }) \\
\mathbf{8 1 , 4}(L=2 \text { jours })\end{array}$ & $\begin{array}{c}66,3(\mathrm{~L}=1 \text { jour }) \\
71,2(\mathrm{~L}=2 \text { jours })\end{array}$ \\
\hline Petit Morin & $\begin{array}{c}6,5 \text { ( } L=1 \text { jour }) \\
61,1 \text { ( } L=2 \text { jours })\end{array}$ & $\begin{array}{l}-22,2(L=1 \text { jour }) \\
50,0(L=2 \text { jours })\end{array}$ & $\begin{array}{c}37,5(\mathrm{~L}=1 \text { jour }) \\
\mathbf{4 8 , 4}(\mathrm{L}=2 \text { jours })\end{array}$ & $\begin{array}{c}39,0(\mathrm{~L}=1 \text { jour }) \\
50,1 \text { ( } \mathrm{L}=2 \text { jours })\end{array}$ \\
\hline
\end{tabular}

De manière générale, on peut noter que la méthode d'assimilation des débits donne des résultats satisfaisants pour les deux modèles, les coefficients de persistance étant toujours significativement supérieurs à 0 , ce qui signifie que la méthode de mise à jour des paramètres par assimilation des débits est plus efficace qu'une prévision « persistante » dite « naïve ». En particulier, plus le délai de prévision augmente, meilleurs sont les coefficients de persistance, ce qui est logique puisque la prévision naïve devient alors moins bonne.

D'autre part, les résultats sans mise à jour des paramètres sont sensiblement équivalents pour les deux modèles, avec un léger avantage pour GR4j. Sur le Petit Morin le coefficient de persistance prend des valeurs faibles voir négatives pour un délai de prévision de 1 jour. Ceci souligne seulement que le modèle en simulation est moins performant sur ce bassin que le modèle de persistance qui pour un délai de 1 jour est très proche des observations. Les résultats avec assimilation des débits, montrent dans ce cas, un léger avantage pour GRHum. La mise à jour des paramètres semble donc plus efficace pour GRHum. En particulier pour le 
Petit Morin pour un délai de 1 jour, on passe d'un coefficient de $-22.2 \%$ à un coefficient de $39 \%$, soit un gain de plus de $60 \%$.

Enfin, nous avons repéré en gras les cas où l'assimilation des débits n'apporte pas d'amélioration en prévision, c'est le cas sur l'Orgeval plus particulièrement. Cela est probablement dû à la taille très réduite du bassin versant $\left(104 \mathrm{~km}^{2}\right)$, qui conduit à des temps de réponse inférieurs à la journée, temps inférieur au délai de prévision.

\subsection{Relation humidités / Etats du système}

Dans un deuxième temps, afin d'optimiser la contrainte sur le système prévu, nous avons étudié les relations entre les états hydriques observés et les états internes des systèmes modélisés par GR4j et GRHum (figure 4 et figure 5). Les figures montrent sur le bassin du Serein les relations obtenues entre les humidités à différentes profondeurs et les états des réservoirs. Pour GRHum, les états des réservoirs sont Ws/Wsmax $=\mathrm{Hs}, \mathrm{Wg} / \mathrm{Wgmax}=\mathrm{Hg}$ et $R / B$, pour GR4j les états internes considérés sont $S / A$ et $R / B$. Pour ce bassin c'est l'humidité profonde qui est la mieux corrélée avec les différents états internes du modèle mais ce n'est pas le cas pour les autres bassins comme nous le verrons à la section suivante. De manière générale, on constate que les relations ont une allure linéaire avec des coefficients de détermination significatifs : ils sont toujours supérieurs à 0,69. Cela justifie donc l'équation linéaire, ainsi que la contrainte sur les humidités utilisée (4).
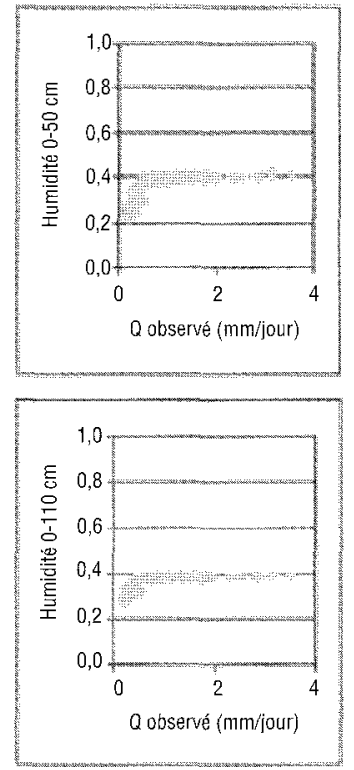
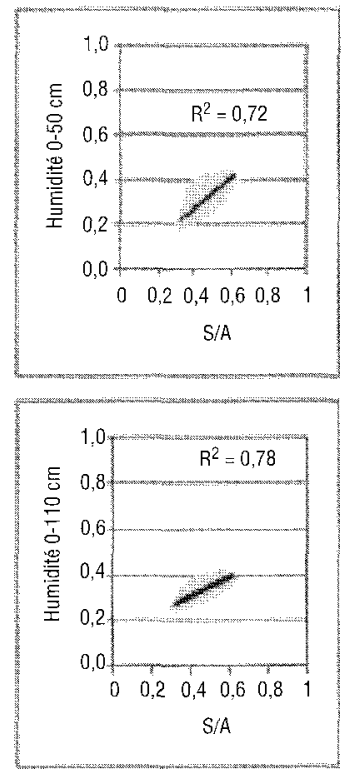
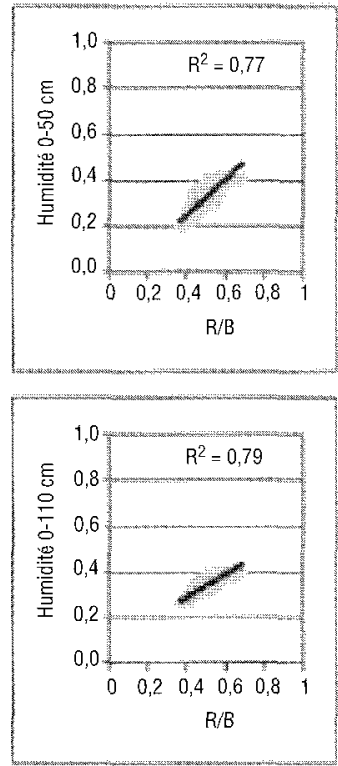

Figure 4 Relation entre humidités (\%) à différentes profondeurs, débit observé et états du système GR4j (exemple du Petit Morin).

Relationship between soil moisture (\%) at different depths, observed streamflow and store contents calculated with GR4j model over the Petit Morin catchment. 

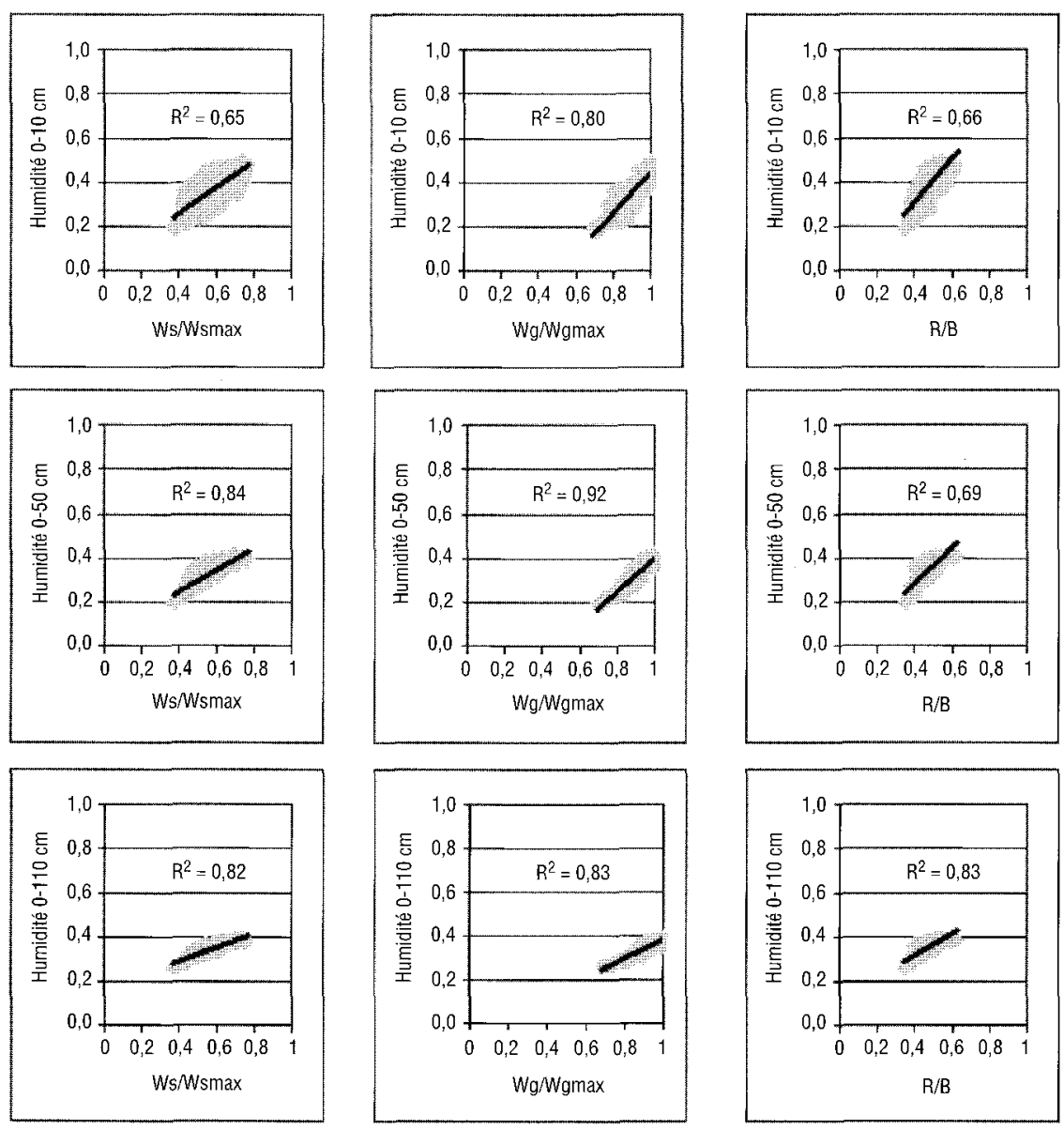

Figure 5 Relation entre humidités (\%) à différentes profondeurs et états du système GRHum (exemple du Petit Morin).

Relationship between soil moisture at different depths and store contents calculated with GRHum model over the Petit Morin catchment.

\subsection{Assimilation des humidités : comparaison des résultats obtenus par les différents modèles}

La méthode a été testée pour les quatre bassins versants pour les périodes tests indiquées dans le tableau 4. Pour chaque bassin versant, les tests effectués en validation sont les suivants:

- On fait varier le paramètre $k$ (cf. équation de contrainte (4)) entre une valeur proche de zéro $(0.001)$ et 20 . Pour $k$ proche de zéro, les humidités ne sont pas utilisées : c'est la méthode de Yang initiale (1993), assimilant uniquement les débits. Plus $\mathrm{k}$ augmente, plus les humidités sont prises en 
compte. On impose enfin $k=500$, pour forcer le système à prendre en compte entièrement les observations d'humidité .

- Les différents états du système sont testés : pour GR4j on teste la relation avec $S / A, R / B$, et $S / A \& R / B$; et pour GRHum, la relation est testée successivement avec $\mathrm{Hs}, \mathrm{Hg}, \mathrm{R} / \mathrm{B}, \mathrm{Hs} \& \mathrm{Hg}$ et $\mathrm{Hs} \& \mathrm{Hg}$ \& R/B. Ces différents états du système représentent les réservoirs superficiels, profonds et gravitaires des modèles, ces réservoirs conceptuels sont interconnectés et dans la procédure d'assimilation la mesure de l'état hydrique à différentes profondeur peut être reliée aux simulations d'humidité (taux de remplissage des réservoirs) ainsi qu'aux variables qui lui sont liées.

- Enfin, une douzaine de sondes TDR par site fournissent les données d'humidité du sol à différentes profondeurs allant de $10 \mathrm{~cm}$ à plus d'un mètre, chacune d'elles est prise en compte dans la méthode.

Parmi tous les résultats obtenus pour les deux modèles GR4j et GRHum, c'est finalement, le couple [état du système - profondeur de mesure de l'humidité] fournissant les meilleurs résultats en terme de persistance et pour un $k$ donné (compris entre 1 et 10 ), qui est retenu pour chaque bassin versant et chaque délai de prévision. Le tableau 6 et la figure 6 présentent ces résultats, que nous analysons par la suite.
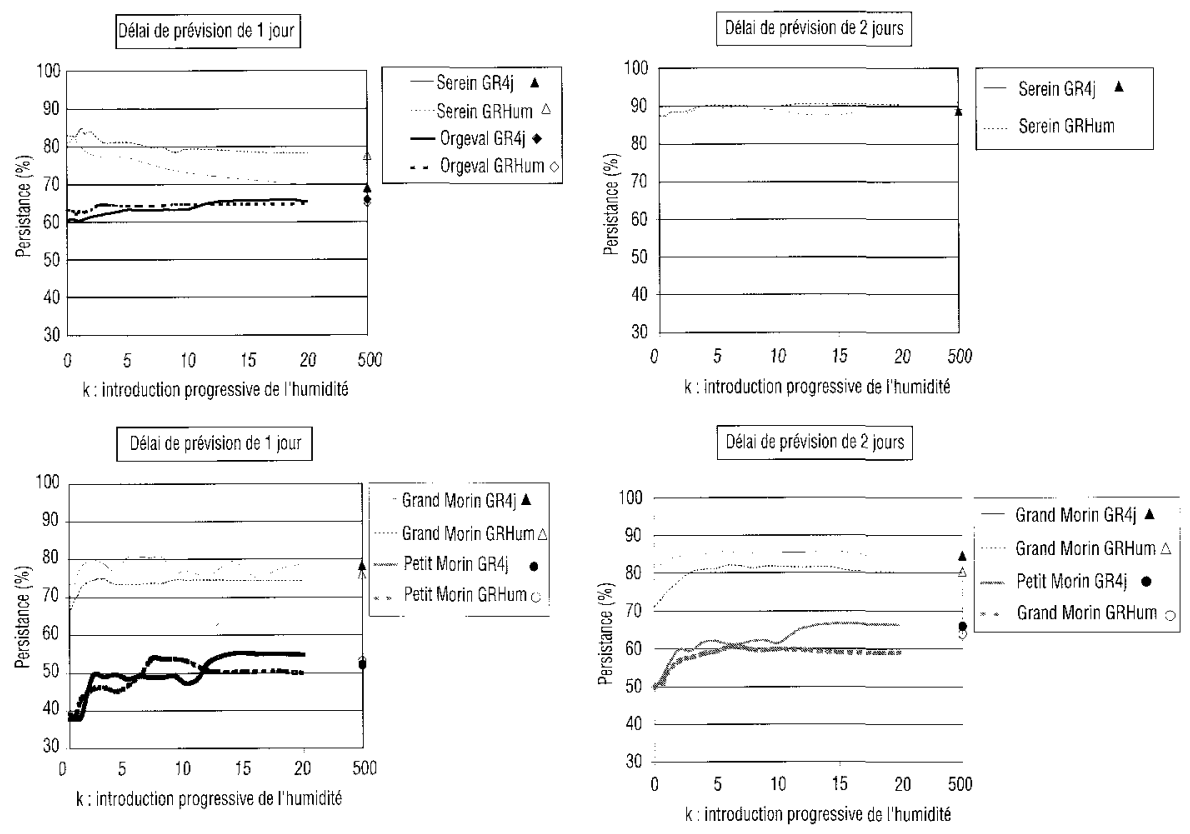

Figure 6 Pour chaque bassin versant, persistance en fonction de $\mathrm{k}$ pour le couple [état du système - profondeur de mesure de l'humidité] qui donne les meilleurs résultats en assimilation des débits et des humidités.

Persistence criterion with gradual introduction of soil moisture information for the best combination [calculated soil moisture - observed soil moisture]. 
Tableau 6 Combinaison [état du système - profondeur de mesure de l'humidité] fournissant les persistances et gains maximaux pour l'assimilation des débits et des humidités comparées à l'assimilation des débits seuls.

Table 6 Combination [calculated soil moisture - observed soil moisture] giving the best improvement of flood forecasting over the studied catchments.

\begin{tabular}{|c|c|c|c|c|c|c|}
\hline & $\begin{array}{c}\text { Délai de } \\
\text { prévision }\end{array}$ & $\begin{array}{c}\text { Modèle } \\
\text { pluie-débit }\end{array}$ & Humidité & $\begin{array}{l}\text { États du } \\
\text { système }\end{array}$ & $\begin{array}{c}\text { Gain / } \\
\text { assim. Q }\end{array}$ & $\begin{array}{c}\text { Persistance } \\
\text { maximale }\end{array}$ \\
\hline \multirow[t]{2}{*}{ Serein } & 1 jour & $\begin{array}{c}\text { GR4j } \\
\text { GRHum }\end{array}$ & $\begin{array}{l}0-110 \mathrm{~cm} \\
0-110 \mathrm{~cm}\end{array}$ & $\begin{array}{c}\text { S/A\&R/B } \\
R / B\end{array}$ & $\begin{array}{l}1,5 \% \\
1,8 \%\end{array}$ & $\begin{array}{l}82,4 \% \\
84,8 \%\end{array}$ \\
\hline & 2 jours & $\begin{array}{c}\text { GR4j } \\
\text { GRHum }\end{array}$ & $\begin{array}{l}0-110 \mathrm{~cm} \\
0-110 \mathrm{~cm}\end{array}$ & $\begin{array}{c}\text { S/A\&R/B } \\
\text { R/B }\end{array}$ & $\begin{array}{l}4,2 \% \\
3,1 \%\end{array}$ & $\begin{array}{l}89,7 \% \\
90,6 \%\end{array}$ \\
\hline Orgeval & 1 jour & $\begin{array}{c}\text { GR4j } \\
\text { GRHum }\end{array}$ & $\begin{array}{l}0-105 \mathrm{~cm} \\
0-30 \mathrm{~cm}\end{array}$ & $\begin{array}{c}\text { R/B } \\
\mathrm{Hs} \& H g\end{array}$ & $\begin{array}{l}5.3 \% \\
1,4 \%\end{array}$ & $\begin{array}{l}65,8 \% \\
64,8 \%\end{array}$ \\
\hline \multirow[t]{2}{*}{ Grand Morin } & 1 jour & $\begin{array}{c}\text { GR4j } \\
\text { GRHum }\end{array}$ & $\begin{array}{l}0-105 \mathrm{~cm} \\
0-10 \mathrm{~cm}\end{array}$ & $\begin{array}{c}\text { S/A\&R/B } \\
\mathrm{Hs} \& H g\end{array}$ & $\begin{array}{r}7,0 \% \\
10,0 \%\end{array}$ & $\begin{array}{l}80,5 \% \\
76,3 \%\end{array}$ \\
\hline & 2 jours & $\begin{array}{c}\text { GR4j } \\
\text { GRHum }\end{array}$ & $\begin{array}{l}0-10 \mathrm{~cm} \\
0-10 \mathrm{~cm}\end{array}$ & $\begin{array}{l}\mathrm{R} / \mathrm{B} \\
\mathrm{R} / \mathrm{B}\end{array}$ & $\begin{array}{r}3,8 \% \\
10,9 \%\end{array}$ & $\begin{array}{l}85,2 \% \\
82,1 \%\end{array}$ \\
\hline Petit Morin & $\begin{array}{l}1 \text { jour } \\
2 \text { jours }\end{array}$ & $\begin{array}{c}\text { GR4j } \\
\text { GRHum } \\
\text { GR4j } \\
\text { GRHum }\end{array}$ & $\begin{array}{l}0-50 \mathrm{~cm} \\
0-10 \mathrm{~cm} \\
0-50 \mathrm{~cm} \\
0-10 \mathrm{~cm}\end{array}$ & $\begin{array}{c}\text { S/A\&R/B } \\
\text { Hs\&Hg\&R/B } \\
\text { S/A\&R/B } \\
\text { Hs\&Hg\&R/B }\end{array}$ & $\begin{array}{l}17,5 \% \\
14,9 \% \\
17,6 \% \\
13,7 \%\end{array}$ & $\begin{array}{l}55,0 \% \\
53,9 \% \\
66,0 \% \\
63,8 \%\end{array}$ \\
\hline
\end{tabular}

Nous pouvons d'ores et déjà noter que pour tous les bassins versants, l'assimilation de l'humidité donne des résultats supérieurs à la méthode initiale assimilant uniquement les débits : tous les critères de persistance augmentent (1,5\% sur le Serein jusqu'à 17,5\% sur le Petit Morin). Cependant, le gain est d'autant plus faible que la méthode initiale d'assimilation des débits était performante, c'est le cas pour le Serein où le critère de persistance est supérieur à $80 \%$. D'autre part, pour l'Orgeval, on n'atteint jamais des persistances aussi bonnes que celles obtenues par simulation simple : sur ce bassin, la méthode d'assimilation (débits seuls ou débits et humidité) en prévision reste non efficace, ce qui est dû à la taille très réduite du bassin versant (104 km²) (cf. § 5.1).

\subsubsection{Influence de la valeur de $k$}

Dans tous les cas étudiés, les critères de persistance varient beaucoup avec les valeurs de $\mathrm{k}$ prises en compte et donnent une idée de l'importance donnée aux mesures d'humidité dans la méthode d'assimilation. Pour $k$ proche de zéro, la contrainte sur les humidités n'est pas prise en compte et on retrouve les résultats de la méthode assimilant uniquement les débits (Tableau 5). Quand k augmente, les courbes des critères de persistance ont des allures assez complexes et semblent stagner après une valeur de $\mathrm{k}$ autour de 10 . Dans ce cas, la méthode d'assimilation ne tient pas entièrement compte de l'information sur les humidités mais tient compte aussi des erreurs sur la mesure et des relations avec les autres variables du modèle. Les oscillations constatées après cette valeur peuvent s'expliquer par le fait que l'objectif de la mise a jour en prévision est ici double: on veut améliorer les simulations des 
débits, tout en améliorant les simulations d'humidité. II se peut alors que le jeu de paramètres retenu améliore la contrainte sur les humidités (éq. (4)) sans améliorer celle sur les débits simulés (éq. (3)). Enfin, les résultats obtenus pour $\mathrm{k}=500$ où la contrainte sur les humidités est très forte, ne sont pas systématiquement les meilleurs. II s'agit ici de l'assimilation par « forçage » où l'on suppose les observations sur les humidités sans erreur. Ce forçage introduit un changement trop brutal dans l'état hydrique modélisé sans tenir compte des relations avec les autres variables du modèle, d'où l'intérêt de faire varier la contrainte sur les humidités dans la méthode d'assimilation.

\subsubsection{Influence de l'état du système}

Les états du système pris en compte dans la relation (2) sont très variables et dépendent du bassin étudié ainsi que du délai de prévision choisi ( 1 ou 2 jours), ce qui permet de tester 7 cas possibles (tableau 6). Pour GR4j, le réservoir de transfert R/B est systématiquement sélectionné, parfois en combinaison avec le réservoir de production S/A (dans 5 cas sur 7 ). Dans un but opérationnel, on pourrait donc imposer d'utiliser systématiquement ce couple. Pour GRHum, les états du système sélectionnés dans la relation (3) sont soit les états hydriques des réservoirs superficiel et global $\mathrm{Hs} \& \mathrm{Hg}$ toujours pris en compte conjointement ( 4 cas sur 7 ) combinés parfois avec le réservoir de transfert $R / B$, soit le réservoir de transfert $R / B$ utilisé seul ( 3 cas sur 7 ). II faut noter que dans ce dernier cas l'humidité assimilée est en général (sauf pour le Grand Morin) I'humidité profonde mieux reliée au réservoir de transfert.

\subsubsection{Influence de la profondeur de mesure d'humidité}

A nouveau, les résultats sont très variables d'un bassin à l'autre et suivant le modèle utilisé. II faut noter cependant, que pour GR4j, ce sont surtout les couches d'humidité profondes qui sont utilisées, en effet, le modèle ne simule pas l'état hydrique de la couche de surface. Les humidités profondes sélectionnées sont comprises entre $0-50 \mathrm{~cm}, 0-105 \mathrm{~cm}$ ou $0-110 \mathrm{~cm}$ dans 6 cas sur 7. II n'y a qu'un cas où l'humidité de surface $(0-10 \mathrm{~cm})$ est sélectionnée : c'est pour le Grand Morin, bassin sur lequel, on le rappelle, les débits sont influencés par la Marne, ce qui rend l'interprétation des résultats plus délicate. Au contraire, pour GRHum, c'est l'humidité des couches superficielles $(0-10 \mathrm{~cm})$ qui est sélectionnée (5 cas sur 7). Cette particularité du modèle GRHum semble indiquer que la prise en compte de deux couches du sol dans la modélisation pluie-débit joue un rôle sur le type de données à assimiler. Cette hypothèse est bien sûr à vérifier notamment lors de l'utilisation de cette méthodologie avec des données satellitales donnant accès seulement à une information sur l'état hydrique de surface.

\subsubsection{Comparaison des résultats des deux modèles GR4j et GRHum}

Se situant dans une optique d'amélioration de la prévision des crues, la comparaison des deux modèles GR4j et GRHum est centrée sur les performances obtenues par ces deux modèles en prévision des débits après remise à jour des paramètres. Le critère de persistance retenu donne une indication sur l'erreur quadratique des débits prévus par rapport aux débits observés. C'est donc uniquement sur les performances liées à ce critère que sont comparés ici les deux modèles. 
Alors que les critères de persistance de la méthode initiale, assimilant uniquement les débits, étaient plutôt en faveur du modèle GRHum (+ 2-3\%), les résultats de l'assimilation conjointe des débits et des humidités sont plutôt en faveur de GR4j (+ 3-4 \%) (Figure 6). II n'y a que pour le Serein que les meilleurs critères de persistance sont obtenus avec GRHum (+ 1-2\%). Ces résultats montrent que dans un contexte de prévision de crues avec assimilation des données d'humidité du sol mesurées in situ, il ne semble pas nécessaire d'utiliser une modélisation de l'interface sol-végétation-atmosphère pour remettre à jour les paramètres du modèle. Le modèle GR4j, avec un seul réservoir de production, semble être aussi apte à tirer parti de cette nouvelle information. Par ailleurs, ces résultats montrent aussi l'intérêt qu'il y a à utiliser une information sur l'état hydrique du sol en prévision de crue il y a toujours un gain en prévision par rapport à l'assimilation seule des débits. Cependant, c'est surtout dans les cas où la méthode initiale n'est pas performante que l'assimilation des états hydriques est vraiment intéressante: on gagne jusqu'à $17,5 \%$ de persistance par rapport à la méthode assimilant uniquement les débits. Dans les autres cas, les gains sont plus modestes (2-11\%). En effet, lorsque les modèles sont déjà performants, les améliorations apportées en prévision par une information supplémentaire sont minimisés. Pour des performances plus modestes en prévision, comme cela serait le cas en prévision avec des pluies non connues les gains pourraient être alors plus importants. Ces conclusions doivent cependant être confirmées avec l'utilisation de scenarii de pluie, sur un nombre plus large de bassins étudiés, un nombre de crues plus important et des données d'humidité utilisées non plus ponctuellement comme c'est le cas ici, mais avec des données satellitales plus représentatives des bassins versants dans leur ensemble. Les données satellitales permettent en effet d'obtenir un indicateur intégré spatialement sur l'ensemble du bassin et qui est utilisé comme donnée d'entrée des modèles globaux (PAUWELS et al., 2002).

\section{6 - CONCLUSIONS}

Les travaux engagés dans le cadre du projet AIMWATER ont permis de mettre au point une méthode d'assimilation des humidités du sol, pour la mise à jour des paramètres du modèle utilisé. Cette méthode s'applique à n'importe quel modèle pluie-débit, pour la prévision de crues. Elle consiste à faire varier le jeu de paramètres du modèle lors de la prévision, en rendant mieux compte des débits et des humidités récemment observés. Un des objectifs de cet article était de pouvoir tester l'intérêt de la méthode mise au point pour plusieurs bassins versants français à l'aide de deux modèles hydrologiques de structure équivalente mais différant dans la description de leur fonction de production. GR4j étant un modèle conceptuel très simple à deux réservoirs et GRHum est un modèle conceptuel couplé à un schéma de surface qui permet de calculer l'évolution de l'humidité du sol superficielle et profonde. A travers cette comparaison nous voulions évaluer l'apport d'une meilleure description des échanges à l'interface sol-végétation-atmosphère vis à vis de l'assimilation de données d'humidité des sols. Les résultats obtenus ont montré que les 
performances des deux modèles sont presque équivalentes avec un léger gain pour l'assimilation des débits seuls avec GRHum ainsi qu'un léger gain pour l'assimilation des humidités avec GR4J. Avec cette méthode de mise à jour par assimilation variationnelle, il ne semble pas nécessaire d'utiliser un modèle décrivant de façon fine les échanges sol-végétation-atmosphère. Cependant, GRHum semble pouvoir assimiler plus facilement les humidités superficielles que profondes, cette particularité du modèle doit être vérifiée avec l'utilisation uniquement de données superficielles en imposant aux deux modèles l'assimilation soit des données mesurées in situ à $10 \mathrm{~cm}$ soit des données issues de l'observation satellitale.

Un autre objectif de l'étude était de fournir aux gestionnaires de barragesréservoirs, co-partenaires de cette recherche, une méthode qui leur permettrait d'améliorer la gestion de leurs ouvrages, dans un contexte de prévision de crues. La mise en œuvre de la mise à jour de la prévision par assimilation des données d'humidités semble être prometteuse pour la prévision de crue et améliore la méthode initiale d'assimilation des débits seuls. En effet, sur les bassins étudiés, la méthodologie testée en validation pour des délais de prévision de 1 ou 2 jours montre des gains significatifs plus particulièrement, quand la méthode initiale utilisant uniquement les débits est peu performante. Le rapport coût efficacité de la méthode notamment après couplage avec des scenarii de pluie doit être testée avant sa mise en œuvre finale dans le cadre des procédures de gestion.

D’autres méthodes sont actuellement en développement, en particulier, une méthode séquentielle basée sur le filtre de Kalman étendu, dont les premiers résultats sur le Serein sont également très encourageants avec des gains de $5 \%$ (AUBERT et al., 2001). II faudra évaluer comparativement les résultats obtenus sur l'ensemble de bassins par rapport à la méthode présentée ici. II restera également à tester la méthode avec des données spatialisées issues de la télédétection plus représentatives de l'état hydrique du bassin. Ces nouvelles données permettent d'accéder à un indicateur de l'état hydrique global d'un bassin versant avec des précisions de $4 \%$ (QUESNEY et al., 2000). Testées sur le bassin de l'Orgeval avec le filtre de Kalman étendu (QUESNEY, 1999) elles améliorent de $10 \%$ le critère de Nash en période de crues.

\section{REMERCIEMENTS}

Cette étude a été réalisée dans le cadre d'un projet européen financé pour 3 ans (Contract $n^{\circ}$ ENV4-CT98-0740 (DG12-ESCY)). Les auteurs veulent remercier ici tous les partenaires du projet (Cemagref, CETP/CNRS, Univ. of Valencia, Univ. Independente, IH Wallingford, Ass. Reg. Ben, IIBRBS) et en particulier l'équipe du Cemagref pour son appui technique pour les mesures d'humidité des sols. 


\section{RÉFÉRENCES BIBLIOGRAPHIQUES}

AMBROISE B. (1999). La dynamique du cycle de l'eau dans un bassin versant Processus, Facteurs, Modèles. Éditions $\mathrm{H}^{\star} \mathrm{G}^{\star} \mathrm{A}$, Bucarest, $200 \mathrm{p}$.

AUBERT D., LOUMAGNE C., WEISSE A., LE HEGARAT-MASCLE S. (2001). Assimilation of Earth Observation data into hydrological models : the sequential method. Remote Sensing and Hydrology Symposium, $5^{\text {th }}$ International Workshop on Application of Remote Sensing in Hydrology, octobre 2001, 8 p, accepted.

DALEY R. (1997). Atmospheric Data Assimilation. J. Meteor. Soc. Japan, 75, 257288.

DEARDORFF, J.W. (1978). Efficient prediction of ground surface temperature and moisture with inclusion of a layer of vegetation. J. Geophys. Res. 83(4), 1889-1903.

EDIJATNO, NASCIMENTO N. O., YANG X., MAKHLOUF Z., MICHEL C. (1999). GR3J : a daily watershed model with three free parameters. Hydrol. Sci. J. 44(2), 263-277.

ENGMAN E.T. (1990). Use of microwave remotely sensed soil moisture in hydrologic modeling. In: Application of Remote Sensing in Hydrology, ed. G.W. Kite \& A. Wankiewicz, 279-292. Proc. Symp. $N^{\circ} 5$, $\mathrm{NHRI}$, Saskatoon, Canada.

IDE K., COURTIER P., GHIL M, LORENC A., 1997. Unified notation for data assimilation : operational, sequential and variational, J. Meteor. Soc., Japan, 75: 181-189.

KITANDINIS P.K. \& BRAS R. (1980). RealTime forecasting with a conceptual hydrologic model, applications and results. Water Resources Research, vol $16, n^{\circ} 6$, pages 1034-1044.

LAMB R. (1999). Calibration of a conceptual rainfall-runoff model for flood frequency estimation by continuous simulation. Water Resour. Res. 35(10), 3103-3114.

LOUMAGNE C., MICHEL C., NORMAND M. (1991). Etat hydrique du sol et prévision des débits. Journal of Hydroloy, 123(1-2), 1-17.

LOUMAGNE C., CHKIR N., NORMAND M., OTTLE C., VIDAL-MADJAR D. (1996).
Introduction of soil/vegetation/atmosphere continuum in a conceptual rainfallrunoff model. Hydrological Sciences Journal, 41(6), 889-902.

LOUMAGNE C., NORMAND M., RIFFARD M., WEISSE A., QUESNEY A., LE HEGARAT-MASCLE S., ALEM F. (2001). Methodology for integration of remote sensing data into hydrological models for reservoir management purposes. Hydrological Sciences Journal, 46(1), 89-102.

OTTLE C., VIDAL-MADJAR D., 1994, Assimilation of soil humidity iverted from infrared remote sensing in an hydrological model over Hapex-Mobilhy region. J. Hydrol., vol.158, 241-264.

OUDIN L., WEISSE A., LOUMAGNE C., LE HEGARAT-MASCLE S. (2001). Assimilation of soil moisture into hydrological models for flood forecasting : a variational approach. Remote Sensing and Hydrology Symposium, $5^{\text {th }}$ International Workshop on Application of Remote Sensing in Hydrology, octobre 2001, 8 , accepted.

PAUWELS V.R.N., R. HOEBEN, N.C.C. VEHOEST, F.P. DE TROCH, P.A TROCH (2002). Improvement of TOPLATS-based discharge predictions through assimilation of ERS-based remotely sensed soil moisture values. Hydrological Processes, 16(5): 995-1013.

PERRIN C. (2000). Vers une amélioration d'un modèle pluie-débit au travers d'une approche comparative. Thèse de Doctorat, Cemagref, INPG, $270 p+$ annexes.

PERRIN C., MICHEL C., ANDRÉASSIAN V. (2001). Does a large number of parameters enhance model performance ? Comparative assessment of common catchment model structures on 429 catchments. Journal of Hydrology, 242, 275-30.

PIETRONIRO A., SOULIS E.D., KOUVEN N., ROTUNNO O., MULLINS D.W. (1993). Using wide swath C-band SAR imagery for basin soil moisture mapping. Can. $J$. Remote Sens., Special Issue, January, 77-82.

REFSGAARD J.C. (1997). Validation and intercomparison of different updating 
procedures for real-time forecasting. Nordic Hydrol., 28, 65-84.

QUESNEY A. (1999). Assimilation de mesures d'humidité de surface dans un modèle hydrologique conceptuel global. Apport de la télédétection radar ERS/SAR. Thèse de Doctorat, Université Paris 7, $179 p+$ annexes.

QUESNEY A., LE HEGARAT-MASCLE S., TACONET O., VIDAL-MADJAR D., WIGNERON J.P., LOUMAGNE C., NORMAND M. (2000). Estimation of watershed soil moisture Index from ERS/SAR data. Remote Sensing of Environnement, 72, 290-303.

WEISSE A., LE HEGARAT-MASCLE S., AUBERT D., LOUMAGNE C. (2001a). Le projet européen AIMWATER : utilisation de l'humidité des sols mesurée par radar embarqué (ERS/SAR) pour la modélisation pluie-débit. Colloque d'Hydrotechnique « Imagerie satellitaire et radar au service de l'eau », Publications S.H.F., Toulouse 20-21 juin 2001, 11-18.

WEISSE A., MICHEL C., AUBERT D., LOUMAGNE C. (2001b). Assimilation of soil moisture in a hydrological model for flood forecasting. Soil-VegetationAtmosphere Transfer Schemes and Large-Scale Hydrological Models. Proc. of the $6^{\text {th }}$ IAHS Scientific Assembly at Maastricht, The Netherlands, July 2001, IAHS Publ. $n^{\circ}$ 270, 249-256.

WETZEL PJ, CHANG J.T, 1987, Concerning the relationship between evaptranspiration and soil moisture, J; Clim. Applic Meteorol, vol. 26, 18-27.

YANG X. (1993). Mise au point d'une méthode d'utilisation d'un modèle pluiedébit conceptuel pour la prévision des crues en temps réel, Thèse de doctorat, Ecole Nationale des Ponts et Chaussées, 350 p. + annexes.

YANG X., MICHEL C. (2000). Flood forecasting with a watershed model: a new method of parameter updating. Hydrol. Sci. J., 45(4), 1-10.

ZEALAND C.M., BURN D.H., SIMONOVIC S.P. (1999). Short term streamflow forecasting using artificial neural networks. J. Hydrol. 214, 32-48. 